\title{
Imbalance acceleration/escape of energetic particles at interplanetary shocks: effect on spectral steepening
}

\author{
Federico Fraschetti $^{a, b, *}$ and Aurelia Balkanski ${ }^{c}$ \\ ${ }^{a}$ Center for Astrophysics | Harvard \& Smithsonian, Cambridge, MA, 02138, USA \\ ${ }^{b}$ Lunar \& Planetary Laboratory, Department of Planetary Sciences, University of Arizona, Tucson, AZ \\ 85721, USA \\ ${ }^{c}$ Harvard College, Cambridge, MA, 02138, USA \\ E-mail: federico.fraschetti@cfa.harvard.edu
}

\begin{abstract}
Growing multispacecraft networks are broadening the opportunity of measuring energy spectra of energetic particles at interplanetary shocks over three decades or more in energy at the same distance (different from $1 \mathrm{AU}$ ) from the Sun. Energetic particles spectra at interplanetary shocks often exhibit a non power law shape, even within two energy decades. We have introduced a 1D transport equation accounting for particle acceleration and escape that allows for escape at all particle energies not only the highest energies and with no free-escape boundary. The diffusion is contributed by self-generated turbulence close to the shock and by pre-existing turbulence far upstream. The momentum spectrum is used to fit the spectra of the 16 GLE events of solar cycle 23.
\end{abstract}

$37^{\text {th }}$ International Cosmic Ray Conference (ICRC 2021)

July 12th-23rd, 2021

Online - Berlin, Germany

\footnotetext{
*Presenter
} 


\section{Introduction}

The steepening of the momentum spectrum of particles accelerated at interplanetary shock waves has renewed theoretical interest due to the opportunity of multi-spacecraft in-situ measurements of a single event at $1 \mathrm{AU}$ and closer to the Sun, e.g., by combining Parker Solar Probe and Solar Orbiter in-situ measurements. Unequivocal deviations from single power-law over at least 2 decades in particle momentum are routinely measured in the suprathermal ions range by multiple detectors at distinct locations [e.g., at $1 \mathrm{AU}, 1]$, or in the time-integrated fluence spectra of energetic particles in Ground Level Enhancements [GLEs, 2]. The spectral bending can result from a number of effects (escape, particle transport, spherical expansion, shock deceleration). In this presentation we outline a recent model [3] for the imbalance between acceleration and escape at shocks. We also present its application to the momentum spectra of the GLEs events recorded during solar cycle 23.

Double power-laws in solar energetic particle (SEP) spectra have been used to fit time-integrated spectra for the past two decades [e.g. 4-6]. Multi-spacecraft in-situ spectra for GLEs are satisfatorily fitted [2] by the empirical broken-power-law Band's model [7]. A number of modifications to the formulation of the Diffusive Shock Acceleration (DSA) model have been introduced to account for spectral steepening. For example, a single power-law momentum spectrum injected near the Sun was shown to be converted into a double-power-law by pure transport effects as a solution of the transport equation if perpendicular diffusion is neglected [8]. Alternatively a combination of 1D radial shock expansion and decrease of the acceleration time scale, with no escape, can produce a spectral break, as calculated by [9].

Protons spectra of the 16 GLE events of solar cycle 23 were well fitted by a log-parabola [10]. In the astrophysical context, $X$-rays photon spectra of blazars [11] are best-fitted by modeling the spectrum of the synchrotron-emitting electrons as a log-parabola. The photon spectrum of the Crab Nebula in the range $\left(10^{-5}-10^{14}\right) \mathrm{eV}$ (from radio to multi-TeV range), was fitted semi-analytically by a log-parabola spectrum of $\mathrm{TeV}$ electrons [12]. The model herein presented can be mapped to a log-parabola.

\section{Outline of the model}

We consider an infinitely planar shock wave. The 1-D steady-state transport equation for the phase-space distribution function for the energetic particles $f(x, p)$, is recast, assuming pitch-angle isotropy in the local plasma frame, as:

$$
U \frac{\partial f(x, p)}{\partial x}=\frac{\partial}{\partial x}\left[\kappa(x, p) \frac{\partial}{\partial x} f(x, p)\right]+\frac{1}{3}\left(\frac{d U}{d x}\right) p \frac{\partial f(x, p)}{\partial p}+S(x, p)-\frac{f(x, p)}{T(x, p)},
$$

where $S(x, p)$ is the source term and the flow speed is written as:

$$
U= \begin{cases}U_{1} & \text { if } x<0, \text { upstream } \\ U_{2} & \text { if } x>0, \text { downstream } .\end{cases}
$$

The catastrophic loss term $f(x, p) / T(x, p)$ represents escape from the shock with a momentumdependent time scale $T(x, p)$. In the modelling of in-situ intensity profiles of interplanetary shocks, 
a momentum-independent free-scape boundary is in tension with the measurements, that show a flattening down to the background level at distinct distances from the shock for distinct particle energy [e.g., 1]. The relation of $T(x, p)$ to the free escape boundary is discussed in detail in [3].

The energetic particle diffusion is contributed by self-generated turbulence close to the shock and by pre-existing turbulence far upstream. The density of the scattering centers decreases linearly and $\kappa$ increases linearly upstream for an Alfvénic self-generated turbulence [13]. Far upstream, the pre-existing interplanetary/interstellar medium turbulence [14] provides a cutoff out at a certain distance from the shock, i.e., $x=\Lambda_{1}$, where $\kappa$ becomes uniform depending only on particle momentum.

We simplify the problem by assuming that $\kappa$ is separable:

$$
\kappa(x, p)= \begin{cases}\kappa_{1}(p) \frac{|x-\epsilon|}{\left|\Lambda_{1}\right|} & \text { if } x<0 \text { and }|x|<\left|\Lambda_{1}\right|, \text { upstream } \\ \kappa_{1}(p) & \text { if } x<0 \text { and }|x|>\left|\Lambda_{1}\right|, \text { far upstream } \\ \kappa_{2}(p) & \text { if } x>0, \text { downstream }\end{cases}
$$

where $\kappa_{i}(p)$ depends only on momentum and $i=1,2$ indicates respectively upstream and downstream, $\epsilon=d_{i} \simeq 10^{7} \mathrm{~cm}$ is the ion inertial length. Here $\kappa \rightarrow \kappa_{1}(p)$ for $x \rightarrow \Lambda_{1}$ is the far upstream diffusion coefficient in the pre-existing turbulence. We assume also the separability of $T$; the upstream $T$ is expected to decrease with the distance from the shock due to the smaller density of scattering centers far from the shock, and higher close to it, whereas the downstream $T$ is expected to be uniform, mimicking the $\kappa$ dependence. Thus,

$$
T(x, p)= \begin{cases}T_{1}(p) \frac{\left|\Lambda_{1}\right|}{|x-\epsilon|} & \text { if } x<0 \text { and }|x|<\left|\Lambda_{1}\right|, \text { upstream } \\ T_{1}(p) & \text { if } x<0 \text { and }|x|>\left|\Lambda_{1}\right|, \text { far upstream } \\ T_{2}(p) & \text { if } x>0, \text { downstream }\end{cases}
$$

\section{Intensity profiles}

The transport Eq. 1 is solved analytically in position space. Far upstream, for $|x-\epsilon| \gg L_{1}(p)$, the intensity profile can be recast as

$$
f(x, p) \rightarrow f_{0}(p) \frac{\Gamma\left(1-v_{1}\right)}{\sqrt{\pi}} \sin \left(\pi v_{1}\right)\left(\frac{|x-\epsilon|}{2 L_{1}(p)}\right)^{v_{1}-1 / 2} \exp \left(-\frac{|x-\epsilon|}{L_{1}(p)}\right), \text { upstream, }
$$

where $f_{0}(p)$ is the phase-space distribution function at the shock $(x=0), \Gamma(z)$ is the gamma function, $v_{1}(p)=\left|\Lambda_{1}\right| U_{1} / 2 \kappa_{1}(p)$ and the upstream escape length is defined as $L_{1}(p) \equiv \sqrt{\kappa_{1}(x, p) T_{1}(x, p)}=$ $\sqrt{\kappa_{1}(p) T_{1}(p)}$. The spatial profile in Eq. 4 has to be compared with the 1D steady state DSA solution with no upstream particle escape. For large distance from the shock, i.e., limit $|x| \gg L_{1}$ (and $\left.|x|<\left|\Lambda_{1}\right|\right), f$ is exponentially suppressed with a roll-over scale $L_{1}(p)$, that plays the role of the diffusion scale $\sim \kappa_{1}(\epsilon, p) / U_{1}$. Thus, particles do not disappear and still spread out to an infinite distance as in DSA, but with an exponentially small amplitude. The DSA upstream profile is recovered at a given location $|\bar{x}| \gg\left|x_{s}\right|$ if the escape time becomes comparable with the acceleration time: $T_{1}(\bar{x}, p) \simeq \kappa_{1}(\bar{x}, p) / U_{1}^{2}$, by using a crude estimate of the acceleration time [15]. 


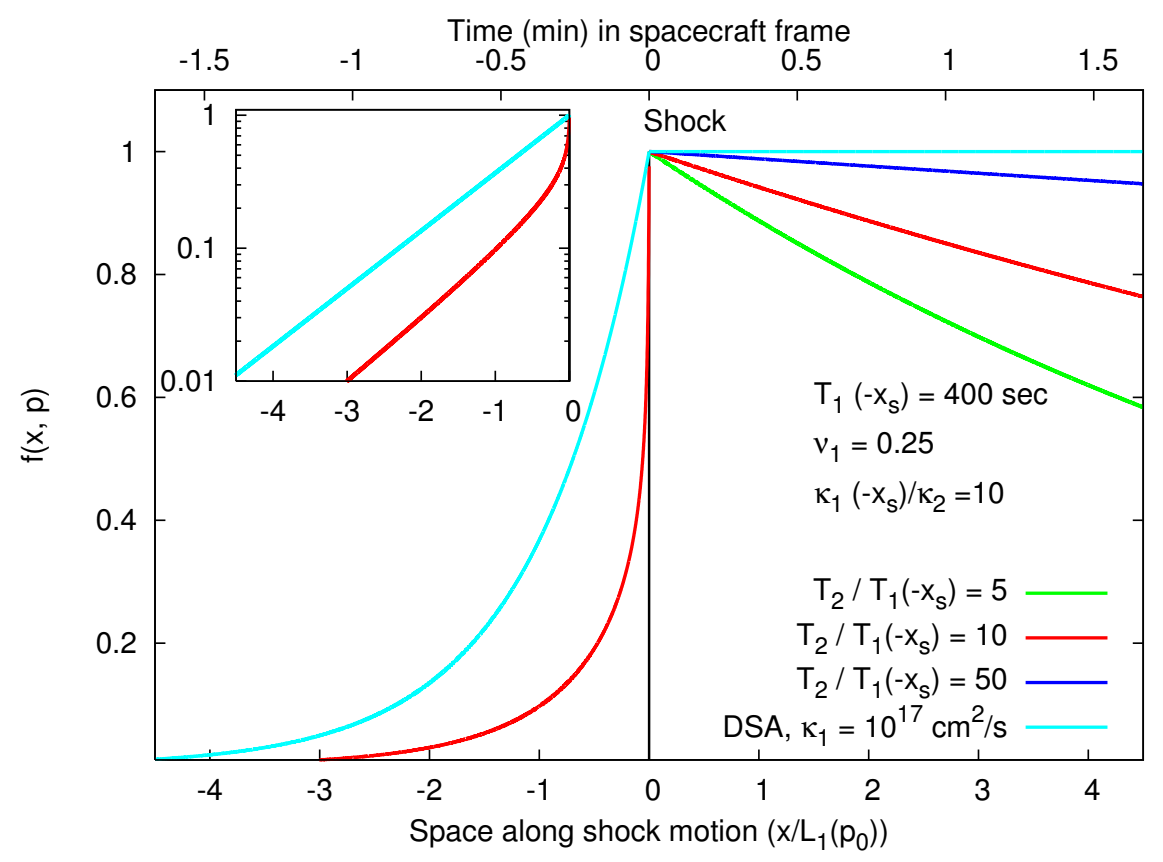

Figure 1: Intensity profiles at momentum $p_{0}$ of energetic particles upstream (Eq. 4) and downstream (Eq. $5)$ of the shock, normalized to the shock value, for fixed $\kappa_{1}\left(-x_{s}, p_{0}\right) / \kappa_{2}\left(p_{0}\right)=10$ and for given ratios $T_{2}\left(p_{0}\right) / T_{1}\left(-x_{s}, p_{0}\right)$. The DSA profile is shown (in cyan) for comparison with $\kappa_{1}^{D S A}=10^{17} \mathrm{~cm}^{2} / \mathrm{s}$. For $\sim 100$ $\mathrm{keV}$ protons, we have used $T_{1}\left(-x_{s}, p_{0}\right)=400 \mathrm{sec}, \kappa_{1}\left(-x_{s}, p_{0}\right)=10^{16} \mathrm{~cm}^{2} / \mathrm{s}$ and $U_{1}=500 \mathrm{~km} / \mathrm{s}$ so that $\kappa_{1}^{D S A} / U_{1}=L_{1}\left(p_{0}\right)=2 \times 10^{9} \mathrm{~cm}$. Both red curves correspond to the case $\kappa_{1}\left(p_{0}\right) / \kappa_{2}\left(p_{0}\right)=T_{2}\left(p_{0}\right) / T_{1}\left(p_{0}\right)=$ 10 so that $L_{1}\left(p_{0}\right)=L_{2}\left(p_{0}\right)$. The shock compression is $r=3$. The vertical black line marks the shock location. The lower $x$-axis gives the distance along the shock normal in units of $L_{1}\left(p_{0}\right)$; the upper $x$-axis gives the time in spacecraft frame (assuming a SW speed $400 \mathrm{~km} / \mathrm{s}$ ). The insert at the top left zooms into the upstream region in logarithmic scale on the $y$-axis.

In the downstream region, by imposing the continuity at the shock, i.e., $f(0, p)=f_{0}(p)$, the limit for $x \rightarrow+\infty$ yields

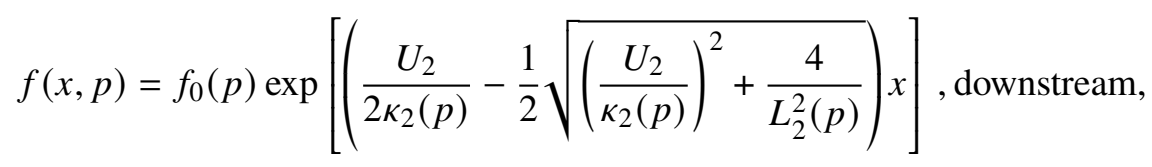

where $L_{2}(p) \equiv \sqrt{\kappa_{2}(p) T_{2}(p)}$ is the downstream escape-length. In the limit of no-escape $\left(T_{2}(p) \gg\right.$ $\kappa_{2}(p) / U_{2}^{2}$ or, equivalently, $\left.L_{2}(p) \gg \kappa_{2}(p) / U_{2}\right)$, the downstream profile tends to the uniform limit $f_{0}(p)$, recovering the DSA solution. Equations 4 and 5 show that the profiles depend on the ratio $\kappa(p) / U L(p)$ (that in the upstream medium coincides with the ratio of the diffusion length to the escape length): if $T(p)$ is very large the DSA solution is retrieved. 


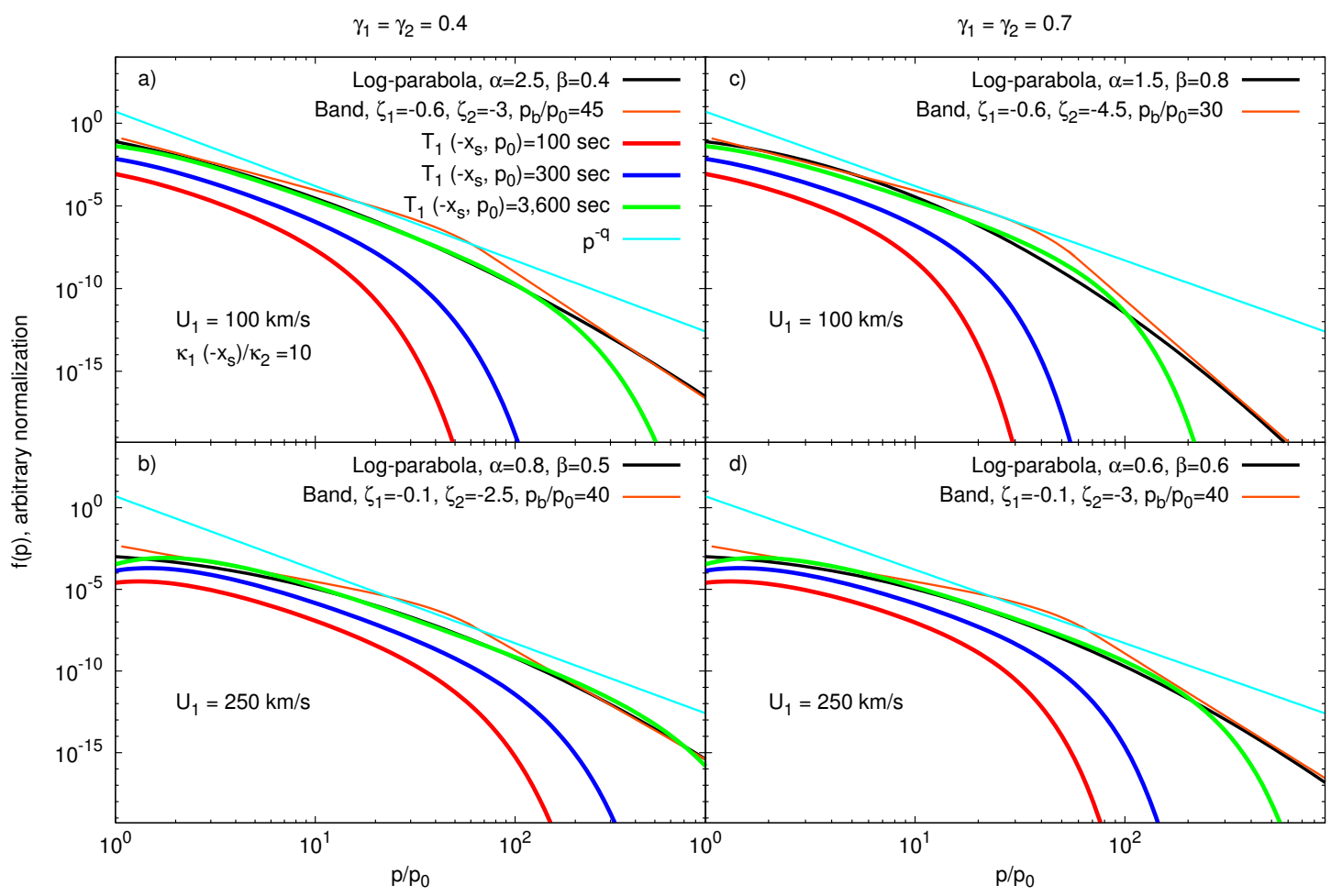

Figure 2: $a$ ): Momentum spectrum in Eq. 8 for distinct values of $T_{1}\left(-x_{s}, p_{0}\right)=100 \mathrm{sec}$ (in red), $300 \mathrm{sec}$ (in blue) and 3,600 sec (in green), compared with the test-particle DSA solution (in cyan), with a log-parabola (in black) reproducing the spectrum in the case $T_{1}\left(-x_{s}, p_{0}\right)=3,600 \mathrm{sec}$ (in this panel up to $p / p_{0} \simeq 150$ that corresponds to $\simeq 4$ decades in particle energy), and with a Band function (in orange) for illustrative purpose with asymptotic slopes fitting the log-parabola. Here $\bar{\kappa}_{1}=10^{19} \mathrm{~cm}^{2} / \mathrm{s},\left|\Lambda_{1}\right|=10^{11} \mathrm{~cm}, r=3, U_{1}=100$ $\mathrm{km} / \mathrm{s}, \delta_{1}=\delta_{2}=1, \gamma_{1}=\gamma_{2}=0.4, \kappa_{1}\left(-x_{s}, p_{0}\right) / \kappa_{2}\left(p_{0}\right)=10$ and in all cases $T_{2}\left(p_{0}\right) / T_{1}\left(-x_{s}, p_{0}\right)=10$ so that $\kappa_{2}(p) / U_{2}^{2} T_{2}(p) \ll 1$. The blue and red curves are multiplied by arbitrary factors, respectively by 0.1 and 0.01 , for readability purposes. $b$ ): Same as $a$ ) with $U_{1}=250 \mathrm{~km} / \mathrm{s} . c$ ): Same as $a$ ) with $\gamma_{1}=\gamma_{2}=0.7 . d$ ): Same as $c$ ) with $U_{1}=250 \mathrm{~km} / \mathrm{s}$.

\section{Momentum spectrum}

The momentum spectrum $f_{0}(p)=f(p)$ is derived from the conservation of the particle number across the shock, i.e., $\int_{-\epsilon}^{+\epsilon} d x U \partial f / \partial x=0$, that applied to Eq. 1 reads:

$$
\left.\left[\kappa(p) \frac{\partial f(x, p)}{\partial x}+\frac{1}{3} U p \frac{\partial f(x, p)}{\partial p}\right]\right|_{-\epsilon} ^{+\epsilon}+\int_{-\epsilon}^{+\epsilon} d x\left[S(x, p)-\frac{f(x, p)}{T(p)}\right]=0 .
$$

We assume a monochromatic source localized at the shock: $S(x, p)=S_{0} \delta(x) \delta\left(p-p_{0}\right)$ and a power-law $p$-dependence of $\kappa$ and $T$ :

$$
\kappa_{i}(p)=\bar{\kappa}_{i}\left(p / p_{0}\right)^{\delta_{i}}, \quad T_{i}(p)=\bar{T}_{i}\left(p / p_{0}\right)^{-\gamma_{i}}, \quad \delta_{1}>\gamma_{1}
$$

where $\bar{\kappa}_{1}=\kappa_{1}\left(\Lambda_{1}, p_{0}\right), \bar{\kappa}_{2}=\kappa_{2}\left(p_{0}\right), \bar{T}_{1}=T_{1}\left(\Lambda_{1}, p_{0}\right), \bar{T}_{2}=T_{2}\left(p_{0}\right)$ and power-law indexes $\delta_{i}>0$, $\gamma_{i}>0$. The condition $\delta_{1}>\gamma_{1}$ ensures that $L_{1}(p)$ increases with $p$. The momentum spectrum can 
be recast as [3]:

$$
f(p) \propto\left(\frac{p}{p_{0}}\right)^{-q} \exp \left[-A_{1}\left(\frac{p}{p_{0}}\right)^{-\delta_{1}}\right] \exp \left[-A_{2}\left(\frac{p}{p_{0}}\right)^{\delta_{2}+\gamma_{2}}\right],
$$

where $q=3 r /(r-1)$ is the DSA test-particle power law in the case of no-escape and

$$
\begin{aligned}
& A_{1}=0.4 \frac{q}{\delta_{1}} \frac{U_{1}}{400 \mathrm{~km} / \mathrm{s}} \frac{\left|\Lambda_{1}\right|}{10^{11} \mathrm{~cm}}\left(\frac{\bar{\kappa}_{1}}{10^{19} \mathrm{~cm}^{2} / \mathrm{s}}\right)^{-1}\left|-5.3+\log \frac{\epsilon / 10^{7} \mathrm{~cm}}{L_{1}\left(p_{0}\right) / 10^{9} \mathrm{~cm}}\right| \\
& A_{2}=6.25 \times 10^{-5} \frac{q}{r\left(\delta_{2}+\gamma_{2}\right)} \frac{\bar{\kappa}_{2}}{10^{15} \mathrm{~cm}^{2} / \mathrm{s}}\left(\frac{U_{2}}{400 \mathrm{~km} / \mathrm{s}}\right)^{-2}\left(\frac{\bar{T}_{2}}{10^{4} \mathrm{sec}}\right)^{-1}
\end{aligned}
$$

where we have used $\bar{T}_{2}=\bar{T}_{1}\left(0, p_{0}\right)$. We note that the expression for $f(p)$ in Eq. 8 does not depend on $\gamma_{1}$.

The width of the peak of the spectrum at low $p$ is controlled by $A_{1}$, namely by $U_{1} \Lambda_{1}\left(\log L_{1}\right) / \bar{\kappa}_{1}$, whereas the drop at large $p$ is controlled by $A_{2}$, namely by $\bar{\kappa}_{2} / U_{2}^{2} \bar{T}_{2}$. The solution in Eq.8 can be mapped into a log-parabola [3]. This model offers a derivation of the log-parabola spectrum complementary to the probability-based derivation used, e.g., in $[11,12]$.

If the spatial diffusion coefficient $\kappa$ is assumed uniform in space both upstream and downstream and discontinuous at the shock only (likewise the escape time $T$ ), the power-law + exponential rollover solution [16] is retrieved as shown in [3].

\section{Application to GLEs of solar cycle 23}

We have used the momentum spectrum in Eq. 8 to model the spectra of the GLE events of solar cycle 23 reported in [2]. An example of two events is shown in Fig. 3.

\section{Conclusion}

We have outlined a steady-state 1D transport model for energetic particles at shocks allowing for a finite acceleration-to-escape time scale ratio upstream at any distance from the shock and at any particle energy and we have shown that it well reproduces the 1-day integrated fluence of the GLEs of solar cycle 23. The upstream diffusion coefficient accounts for the self-generated turbulence due to streaming ions and for the far upstream turbulence. The intensity profiles and momentum spectra of energetic particles remarkably differ from the DSA solution. In particular, the 1D momentum spectrum has the form of the product of a power law and two exponentials. In summary, the power-law scaling resulting from the particle energization increasing at higher energy is extended herein to a more general scaling that results from allowing particle escape at any energy/distance from the shock, not only at the highest energy.

\section{Acknowledgments}

FF was supported, in part, by NSF under grant 1850774, by NASA under Grants 80NSSC18K1213 and 80NSSC20K1283. 

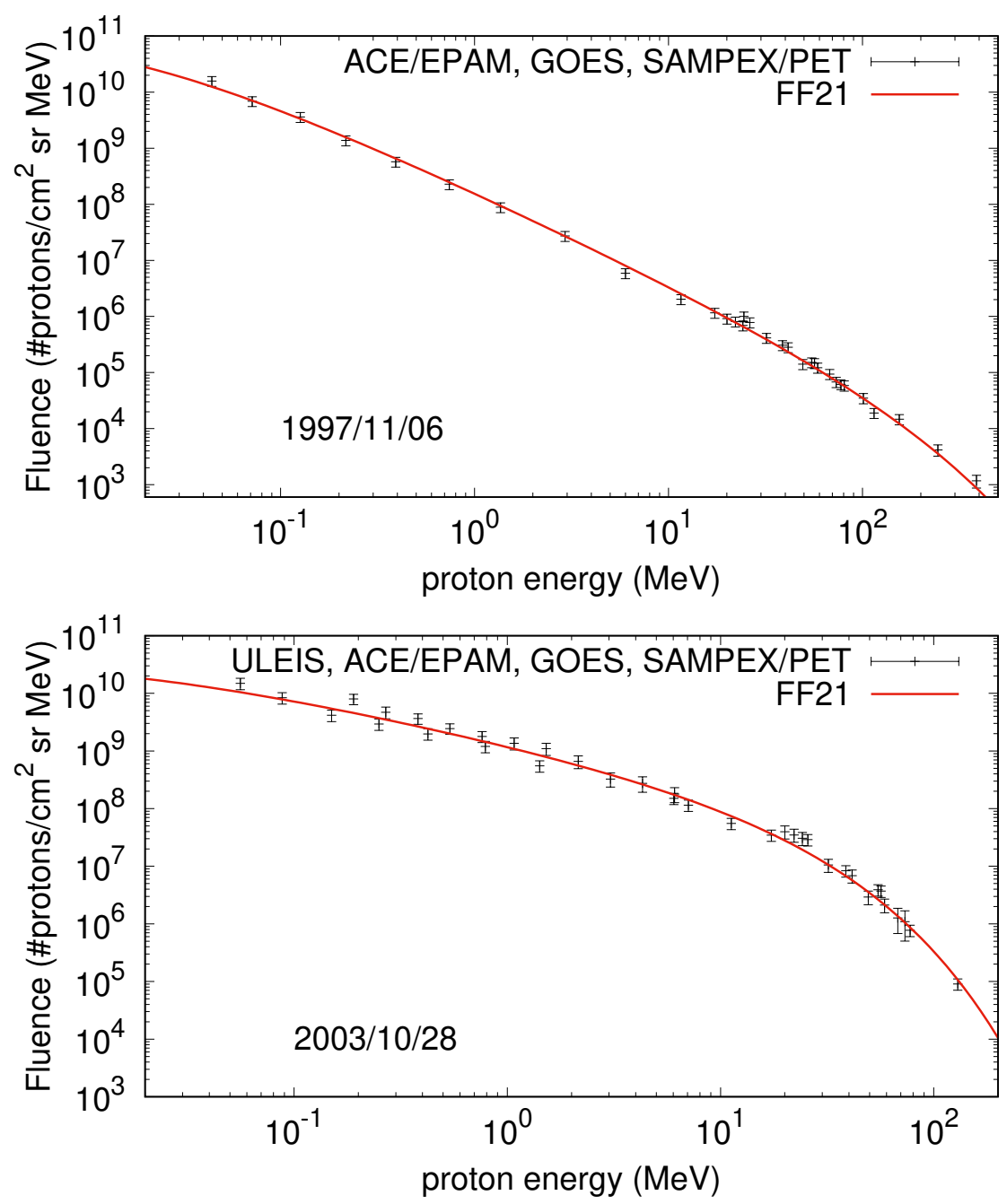

Figure 3: Top: Fluence 1-day integrated spectrum as measured by ACE/EPAM, GOES-11 and SAMPEXPET during the event on November $06^{\text {th }} 1997$ fitted with the spectrum in Eq.8 (red line). For $\delta_{1}=\delta_{2}=1$ and $\gamma_{2}=0.4$, the best-fit values are $q / 2=1.672+/-0.033, A_{1}=0.226+/-0.032$ and $A_{2}=0.0366+/-0.0075$ $\left(\chi^{2} /\right.$ ndf $\left.=0.725\right)$. Top: Same as top for the event of October $28^{\text {th }} 2003$ with best-fit values $q / 2=$ $0.839+/-0.052, A_{1}=0.119+/-0.046$ and $A_{2}=0.183+/-0.013\left(\chi^{2} / \mathrm{ndf}=1.29\right)$.

\section{References}

[1] D. Lario, L. Berger, R.B. Decker, R.F. Wimmer-Schweingruber, I. Wilson, L. B., J. Giacalone et al., Evolution of the Suprathermal Proton Population at Interplanetary Shocks, 158 (2019) 12.

[2] R.A. Mewaldt, M.D. Looper, C.M.S. Cohen, D.K. Haggerty, A.W. Labrador, R.A. Leske et al., Energy Spectra, Composition, and Other Properties of Ground-Level Events During Solar Cycle 23, 171 (2012) 97.

[3] F. Fraschetti, Effect of Acceleration and Escape of Energetic Particles on Spectral Steepening at Shocks, 909 (2021) 42 [2012.12073]. 
[4] G.M. Mason, M.E. Wiedenbeck, J.A. Miller, J.E. Mazur, E.R. Christian, C.M.S. Cohen et al., Spectral Properties of He and Heavy Ions in ${ }^{3}$ He-rich Solar Flares, 574 (2002) 1039.

[5] R.A. Mewaldt, C.M.S. Cohen, G.M. Mason, A.W. Labrador, M.L. Looper, D.E. Haggerty et al., Solar Energetic Particle Spectral Breaks, in The Physics of Collisionless Shocks: 4th Annual IGPP International Astrophysics Conference, G. Li, G.P. Zank and C.T. Russell, eds., vol. 781 of American Institute of Physics Conference Series, pp. 227-232, Aug., 2005, DOI.

[6] M.I. Desai, G.M. Mason, M.A. Dayeh, R.W. Ebert, D.J. McComas, G. Li et al., Spectral Properties of Large Gradual Solar Energetic Particle Events. II. Systematic Q/M Dependence of Heavy Ion Spectral Breaks, 828 (2016) 106 [1605 . 03922].

[7] D. Band, J. Matteson, L. Ford, B. Schaefer, D. Palmer, B. Teegarden et al., BATSE observations of gamma-ray burst spectra. I - Spectral diversity, 413 (1993) 281.

[8] G. Li and M.A. Lee, Scatter-dominated Interplanetary Transport of Solar Energetic Particles in Large Gradual Events and the Formation of Double Power-law Differential Fluence Spectra of Ground-level Events during Solar Cycle 23, 810 (2015) 82.

[9] L.O. Drury, Escaping the accelerator: how, when and in what numbers do cosmic rays get out of supernova remnants?, 415 (2011) 1807 [1009.4799].

[10] C. Zhou, F. Fraschetti, J.J. Drake and M. Pohl, Spectral curvature of shock-accelerated particles in solar cycle 23, Research Notes of the AAS 2 (2018) 145.

[11] E. Massaro, M. Perri, P. Giommi and R. Nesci, Log-parabolic spectra and particle acceleration in the BL Lac object Mkn 421: Spectral analysis of the complete BeppoSAX wide band X-ray data set, 413 (2004) 489 [astro-ph/0312260].

[12] F. Fraschetti and M. Pohl, Particle acceleration model for the broad-band baseline spectrum of the Crab nebula, 471 (2017) 4856 [1702 .00816].

[13] A.R. Bell, The acceleration of cosmic rays in shock fronts. I, 182 (1978) 147.

[14] J.W. Armstrong, B.J. Rickett and S.R. Spangler, Electron density power spectrum in the local interstellar medium, 443 (1995) 209.

[15] W.I. Axford, Late paper: Acceleration of cosmic rays by shock waves, in ESA Special Publication, S.A. Colgate, ed., vol. 161 of ESA Special Publication, p. 425, Nov., 1981.

[16] D.C. Ellison and R. Ramaty, Shock acceleration of electrons and ions in solar flares, 298 (1985) 400. 